\title{
Clinical significance of plasma metastin level in pancreatic cancer patients
}

\author{
FUMIHIKO KATAGIRI $^{1 *}$, KAZUYUKI NAGAI ${ }^{2 *}$, ATSUSHI KIDA $^{2}$, KENJI TOMITA $^{3}$, \\ SHINYA OISHI $^{3}$, MASAHARU TAKEYAMA ${ }^{1}$, RYUICHIRO DOI $^{2}$ and NOBUTAKA FUJII ${ }^{3}$ \\ ${ }^{1}$ Department of Clinical Pharmacy, Oita University Hospital, Oita; \\ ${ }^{2}$ Division of Hepato-Biliary-Pancreatic Surgery and Transplantation, Department of Surgery, \\ Graduate School of Medicine; ${ }^{3}$ Graduate School of Pharmaceutical Sciences, Kyoto University, Kyoto, Japan
}

Received October 7, 2008; Accepted November 24, 2008

DOI: $10.3892 /$ or_00000289

\begin{abstract}
Metastin, which is a 54-residue peptide coded by KiSS-1 gene, is an endogenous ligand to a G-protein-coupled receptor GPR54. Metastin suppresses a malignant tumor to metastasize and regulates secretion of gonadotropine releasing hormone. Physiological action of metastin has been focused on in oncology. It is reported that less KiSS-l gene and more hOT7T175 gene which codes GPR54 are expressed in pancreatic cancers than in normal pancreatic tissues; however, there is no study that investigates the relationship between clinicopathological characteristics and plasma metastin concentration in pancreatic cancer patients. The purpose of this study was to investigate the relationship between plasma metastin-like immunoreactive substance (LI) levels and clinical characteristics in pancreatic cancer patients. Thirtythree patients with pathologically confirmed pancreatic cancer before or just after treatments and 24 healthy volunteers were included in the study. Patients were grouped according to the International Union Against Cancer TNM classification. Plasma metastin-LI was measured by enzyme immunoassay. The plasma metastin-LI levels of cancer patients were significantly higher when compared with healthy volunteers. Significant relationship was not found between the plasma metastin-LI levels and the clinicopathological factors such as tumor size, invasion, lymph node metastasis and distant metastasis. The plasma metastin levels may be a significant biomarker to predict the presence of pancreatic cancer and could be used in pancreatic cancer screening.
\end{abstract}

Correspondence to: Dr Ryuichiro Doi, Department of HepatoBiliary-Pancreatic Surgery and Transplantation, Kyoto University, 54 Shogoin-kawaharacho, Sakyo, Kyoto 606-0857, Japan E-mail: doir@kuhp.kyoto-u.ac.jp

${ }^{*}$ Contributed equally

Key words: metastin, pancreatic neoplasm, metastasis

\section{Introduction}

Metastasis is the most life-threatening complication of solid tumors. When surgical intervention achieves complete resection of the primary neoplasm, the patient can be cured unless there are micro-metastases at distant sites. Tumor metastasis is a complex multi-step process, involving invasion of primary cancer cells into local tissue, angioinvasion, cell migration, reimplantation and proliferation. In addition, previous data have demonstrated the importance of cell surface receptors in determining the likelihood and location of metastatic tumor implants (1). Therefore, it is clear that inhibition of metastasis would result in improved clinical outcome for most cancers.

Metastin has been identified as the endogenous ligand for an orphan heptahelical receptor (hOT7T175, GPR54) that couples primarily to $\mathrm{Gp} / 11(2-5)$. The binding of metastin to its receptor has been shown to inhibit chemotaxis in vitro, to enhance the expression and activity of focal adhesion kinase and to inhibit the ability of metastin receptor-overexpressing melanoma cells to metastasize in vivo (6).

Metastin is expressed in normal organs such as placenta, testis, liver, small intestine and pancreas (6). GPR54 is overexpressed in melanoma (2), hepatocellular (3), thyroid (4), breast (5), esophageal (7), bladder (8) and pancreatic cancer tissues (9). However, the effect of metastin differs according to the type of cancer; lack of KiSS-1 leads to poor progression of bladder cancer and poor outcome (8); lack of KiSS-1 and hOT7T175 predicts lymph node metastasis of esophageal cancer (7); KiSS-1 is overexpressed in advanced breast cancer (5); and overexpression of KiSS-1 and hOT7T175 causes progression of liver cancer (3).

In pancreatic cancer tissue, KiSS-1 is less expressed and hOT7T175 is more expressed when compared with those in normal pancreas tissue (9). However, there are no studies on the relationship between plasma metastin levels and clinical characteristics or outcome of pancreatic cancer. The purpose of this study was to investigate the relationship between plasma metastin-LI level and clinical characteristics in pancreatic cancer patients. 


\section{Patients and methods}

Patients and healthy volunteers. The study design was approved by the Ethics Committees of Oita Medical University and Kyoto University Hospital. All patients and healthy volunteers included in this study received information on the design and scientific purposes of the study and gave written informed consent.

Thirty-three patients with pathologically confirmed pancreatic cancer (17 female, 16 male, aged 42-79 years) were included in the study. Blood sampling was performed in the morning, before the patients were treated by chemotherapy, radiation therapy or surgery. While surgical resection was performed for the patients with resectable cancer, chemotherapy was performed for the patients with locally advanced cancer and/or distant organ metastasis. The patients were evaluated according to the UICC (International Union Against Cancer) TNM classification 6th edition (10) based on the pathological findings.

Twenty-four healthy volunteers (12 female, 12 male) (aged 46-83 years) were included in the study. The subjects did not receive any medication 1 month before and during the study.

Materials. Synthetic human metastin-10 was purchased from the Peptide Institute (Osaka, Japan). Antiserum to metastin-10 (G-048-56) was purchased from Phoenix Pharmaceuticals (Belmont, CA, USA). Goat affinity-purified antibody to rabbit IgGs (whole molecule) (55641) was purchased from ICN Pharmaceuticals (Aurora, OH, USA). MUG and EMCsuccinimide were purchased from Sigma (St. Louis, MO, USA). ß-Galactosidase and aprotinin (Trasylol) were purchased from Boehringer Mannheim (Mannheim, Germany) and Bayer (Leverkusen, Germany), respectively. All other reagents were analytical grade reagents from commercial sources.

EIA procedure for metastin-LI. Peptide levels in plasma were measured using highly sensitive EIAs for metastin-LI, that was developed by us and has been described previously (11). Assays were performed using a delayed-addition method. Separation of bound and free antigens was performed on anti-rabbit IgG-coated immunoplates (Nunc-Immuno Module Maxisorp F8; InterMed, Denmark). Human metastin-10 was conjugated with $B$-D-galactosidase using EMC-succimide according to the methods previously reported (12). The EIAs for metastin-LI were specific and highly sensitive (11).

Preparation of plasma extracts. Blood samples were placed in chilled tubes containing aprotinin $(500 \mathrm{KIU} / \mathrm{ml})$ and EDTA $(1.2 \mathrm{mg} / \mathrm{ml})$ and centrifuged immediately. Each plasma aliquot was diluted 5-fold with $4 \%$ acetic acid ( $\mathrm{pH} \mathrm{4.0)}$ and loaded onto a C18 reversed-phase cartridge (Sep-Pak C18; Millipore, Milford, MA, USA). After washing with 4\% acetic acid, peptides in plasma were eluted with $70 \%$ acetonitrile in $0.5 \%$ acetic acid ( $\mathrm{pH} 4.0)$. The eluted samples were concentrated by spin-vacuum evaporation, lyophilized and stored at $40^{\circ} \mathrm{C}$ until assayed.

Statistical analysis. The results are expressed as box and whisker plot (highest, third quartile, median, first quartile and
Table I. Clinical and histological characteristics of the patients with pancreatic cancer.

\begin{tabular}{|c|c|}
\hline Patients, $\mathrm{n}$ & 33 \\
\hline \multicolumn{2}{|l|}{ Gender, n (\%) } \\
\hline Male & $17(51.5)$ \\
\hline Female & $16(48.5)$ \\
\hline Mean age (SD), years & $60.9(9.7)$ \\
\hline \multicolumn{2}{|l|}{ TNM classification, $\mathrm{n}(\%)$} \\
\hline \multicolumn{2}{|l|}{ T-primary tumor } \\
\hline $\mathrm{T} 2$ & $3(9.1)$ \\
\hline $\mathrm{T} 3$ & $22(66.7)$ \\
\hline $\mathrm{T} 4$ & $8(24.2)$ \\
\hline \multicolumn{2}{|l|}{ N-regional lymph node } \\
\hline $\mathrm{NX}$ & $3(9.1)$ \\
\hline NO & $7(21.2)$ \\
\hline N1 & $23(69.7)$ \\
\hline \multicolumn{2}{|l|}{ M-distant metastasis } \\
\hline M0 & $19(57.6)$ \\
\hline M1 & $14(42.4)$ \\
\hline \multicolumn{2}{|l|}{ Stage, n (\%) } \\
\hline $1 \mathrm{~B}$ & $1(3.0)$ \\
\hline $2 \mathrm{~A}$ & $5(15.2)$ \\
\hline $2 \mathrm{~B}$ & $12(36.4)$ \\
\hline 3 & $1(3.9)$ \\
\hline 4 & $14(42.4)$ \\
\hline \multicolumn{2}{|l|}{ Tumor invasion to, $\mathrm{n}(\%)^{\mathrm{a}}$} \\
\hline Artery & $11(33.3)$ \\
\hline Portal vein & $16(48.5)$ \\
\hline Nerve & $19(57.6)$ \\
\hline Other organs & $2(6.1)$ \\
\hline \multicolumn{2}{|l|}{ Distant metastasis to, $\mathrm{n}(\%)^{\mathrm{b}}$} \\
\hline Liver & $8(24.2)$ \\
\hline Lymph node & $8(24.2)$ \\
\hline Peritoneum & $4(12.1)$ \\
\hline \multicolumn{2}{|l|}{ Surgery, n (\%) } \\
\hline Resectable & $13(39.4)$ \\
\hline Unresectable & $17(51.5)$ \\
\hline Neoadjuvant chemotherapy + resection & $3(9.1)$ \\
\hline
\end{tabular}

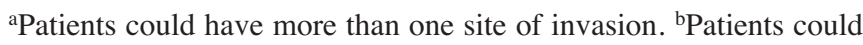
have more than one site of metastasis.

lowest value). Comparison of the results was made by Mann-Whitney $\mathrm{U}$ test. $\mathrm{P}<0.05$ indicates statistical significance.

\section{Results}

A total of 33 pancreatic cancer patients and 24 healthy volunteers were included in the study (Table I). There was no significant difference in ages between pancreatic cancer patients and healthy volunteers $(\mathrm{P}=0.698)$.

In pancreatic cancer patients, as well as in healthy volunteers, there was no significant correlation between plasma metastin-LI levels and age $(\mathrm{P}=0.204$ and 0.667$)$ 


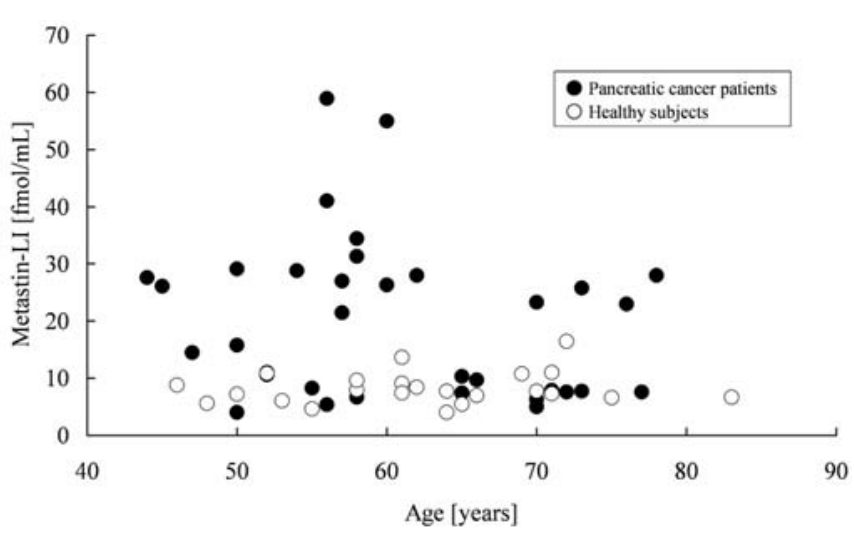

Figure 1. The relationship between age and plasma metastin-LI levels. [ Pancreatic cancer patients $(n=33)$ and $\bigcirc$ healthy volunteers $(n=24)]$.

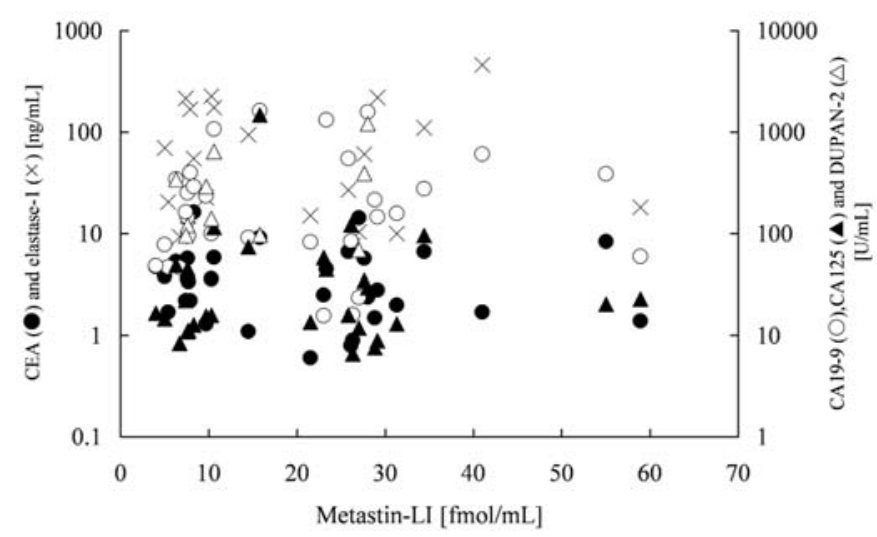

Figure 2. The relationship between some tumor markers and plasma metastin-LI levels. [○ CEA $(n=32), \circ$ CA19-9 $(n=32), \Delta$ CA125 $(n=29)$, $\triangle$ DUPAN2 $(n=11), X$ elastase-1 $(n=25)]$.

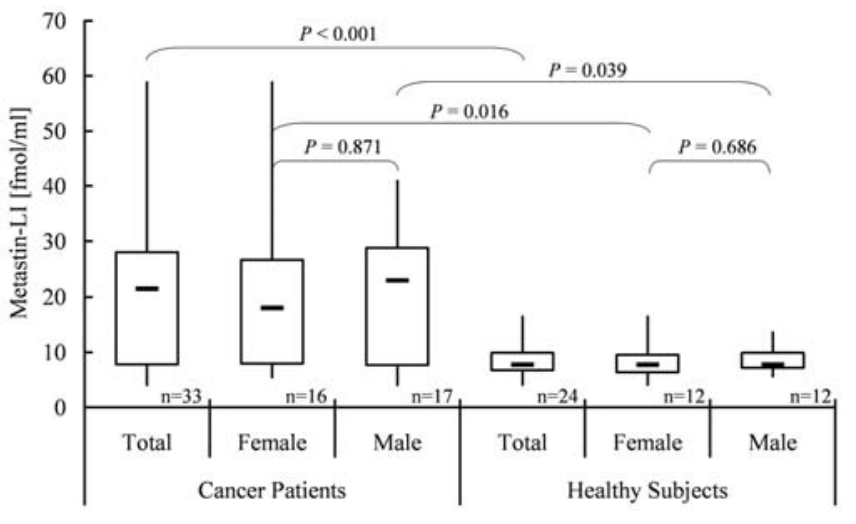

Figure 3. Comparison of plasma metastin-LI levels between pancreatic cancer patients and healthy volunteers. Each value represents the box and whisker plot (highest, third quartile, median, first quartile and lowest value). ${ }^{*} \mathrm{P}<0.05$, significantly different compared to plasma metastin-LI levels in healthy volunteers.

(Fig. 1). The plasma metastin-LI levels also showed no significant correlation with tumor markers [carcinoembryonic antigen (CEA), carbohydrate antigen 19-9 (CA19-9), CA125, pancreatic cancer-associated antigen (DUPAN-2), elastase-1] $(\mathrm{P}=0.788,0.721,0.767,0.155,0.420)$ (Fig. 2).

There were no significant differences in the plasma metastin-LI levels between female and male in healthy volunteers and pancreatic cancer patients. The plasma metastin-LI levels in pancreatic cancer patients were significantly higher than those in healthy volunteers (Fig. 3). Although the plasma metastin-LI levels in patients with T2 tumor were not significantly different from those in healthy volunteers $(\mathrm{P}=0.855)$, the plasma metastin-LI levels in patients of T3, T4, N0, N1, M0 and M1 group were significantly
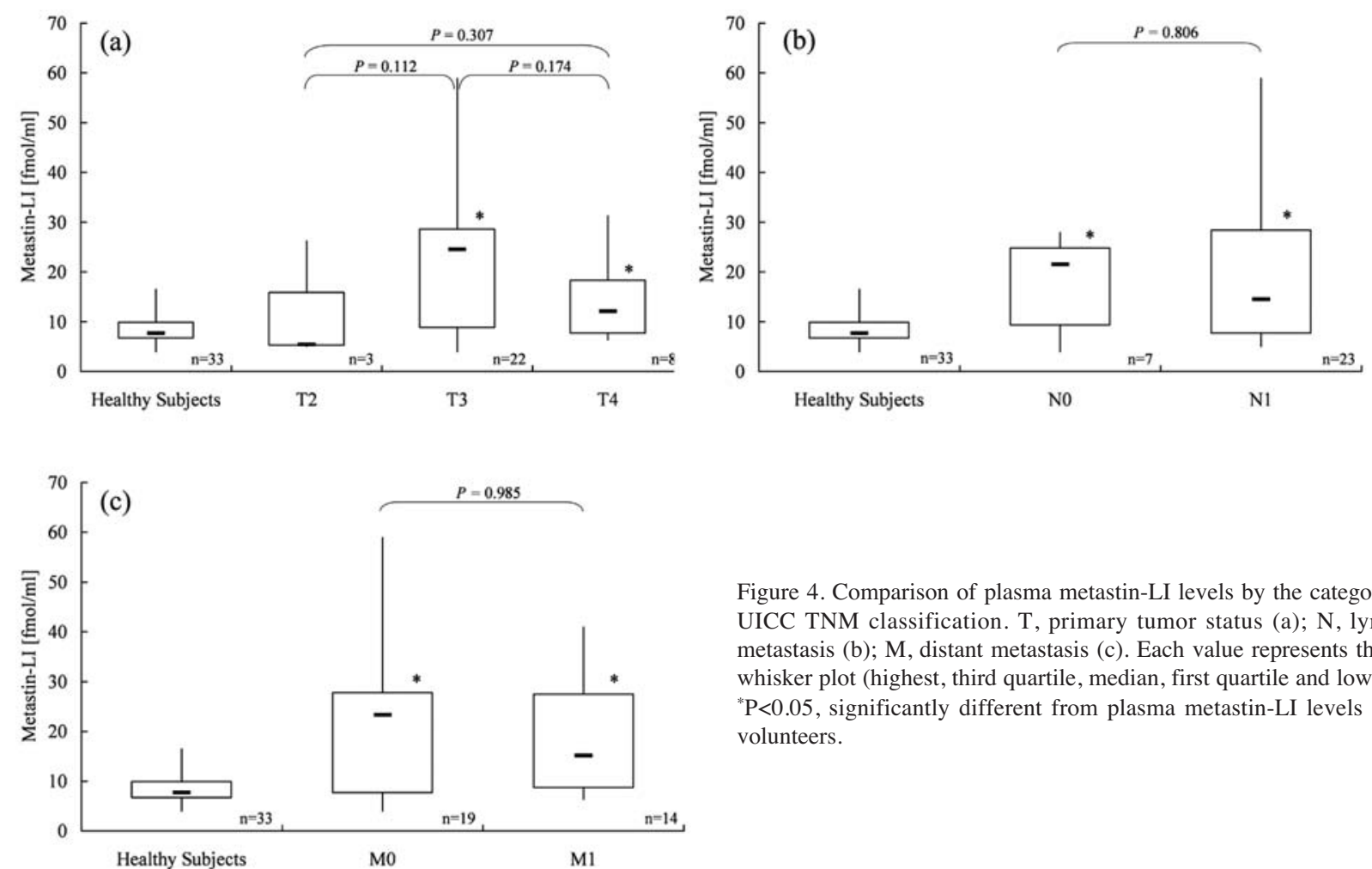

Figure 4. Comparison of plasma metastin-LI levels by the categories of the UICC TNM classification. T, primary tumor status (a); N, lymph node metastasis (b); M, distant metastasis (c). Each value represents the box and whisker plot (highest, third quartile, median, first quartile and lowest value). ${ }^{*} \mathrm{P}<0.05$, significantly different from plasma metastin-LI levels in healthy volunteers. 


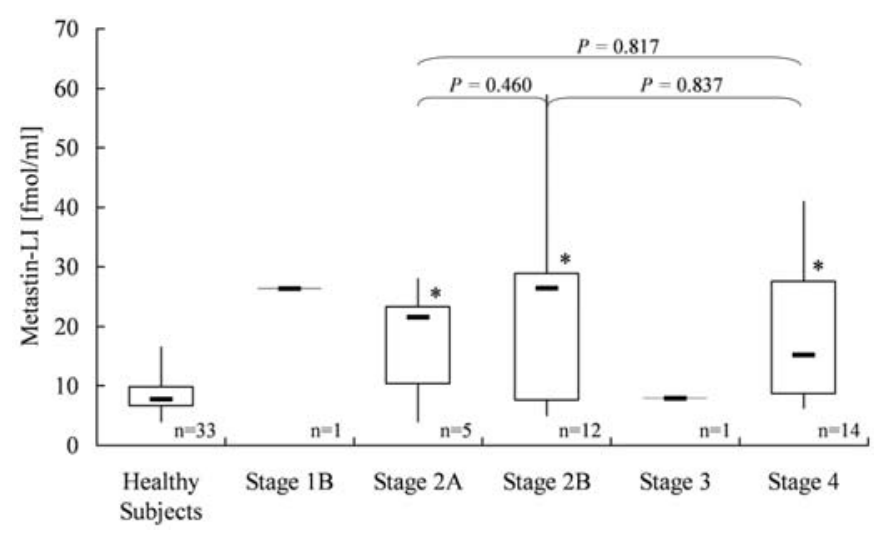

Figure 5. Comparison of plasma metastin-LI levels by the UICC stage. Each value represents the box and whisker plot (highest, third quartile, median, first quartile and lowest value). ${ }^{*} \mathrm{P}<0.05$, significantly different from plasma metastin-LI levels in healthy volunteers.

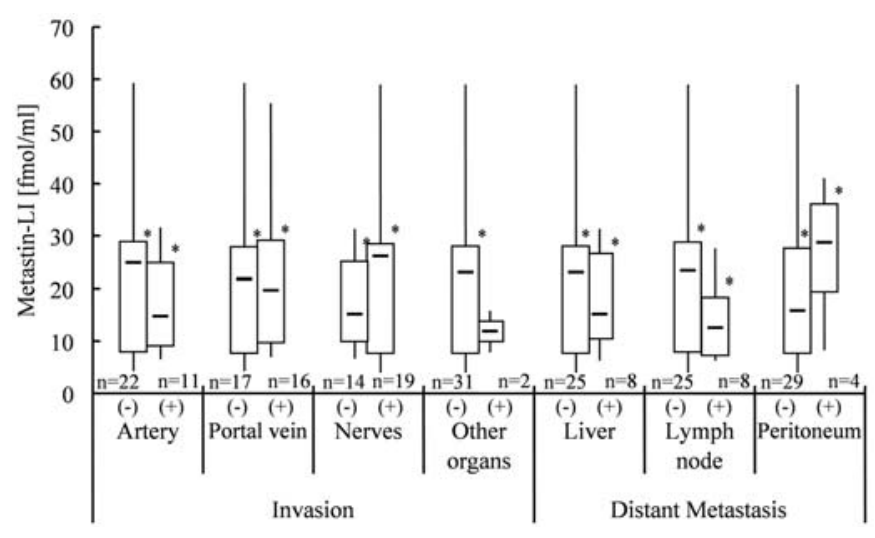

Figure 6. Comparison of plasma metastin-LI levels by the status of invasion or metastasis stage. Each value represents the box and whisker plot (highest, third quartile, median, first quartile and lowest value). ${ }^{*} \mathrm{P}<0.05$, significantly different from plasma metastin-LI levels in healthy volunteers.

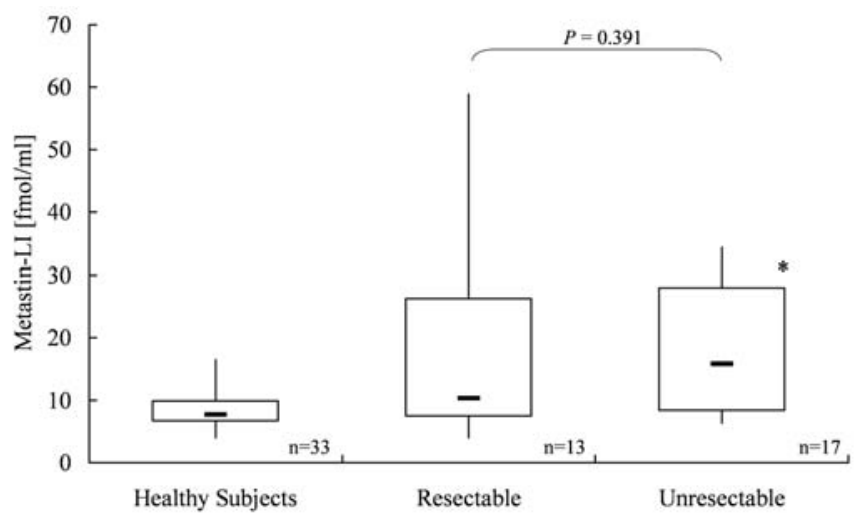

Figure 7. Comparison of plasma metastin-LI levels by the resectability. Each value represents the box and whisker plot (highest, third quartile, median, first quartile and lowest value). ${ }^{*} \mathrm{P}<0.05$, significantly different from plasma metastin-LI levels in healthy volunteers.

higher than those in healthy volunteers $(\mathrm{P}<0.001,0.017,0.009$, $<0.001,<0.001,<0.001)$.
Plasma metastin-LI levels in cancer patients did not differ by categories of the UICC TNM classification (Fig. 4a-c). Plasma metastin-LI levels did not significantly differ by UICC stage (Fig. 5). The plasma metastin-LI levels in patients of stage $2 \mathrm{~A}, 2 \mathrm{~B}$ and 3 groups were significantly higher than those in healthy volunteers $(\mathrm{P}=0.027,0.005,<0.001)$.

Plasma metastin-LI levels did not differ significantly by the status of invasion or metastasis $[\mathrm{P}=0.504$ (artery), 0.313 (portal vain), 0.702 (nerves), 0.983 (liver), 0.179 (lymph node), 0.225 (peritoneum)] (Fig. 6).

The plasma metastin-LI levels were not significantly different between resectable and unresectable patients (Fig. 7). Although the plasma metastin-LI levels in resectable patients were not significantly different from those in healthy volunteers $(\mathrm{P}=0.166)$, those in unresectable patients were significantly higher than in healthy volunteers $(\mathrm{P}=0.001)$.

\section{Discussion}

Metastin is known to have a stimulating effect on gonadotropin secretion (13) and is related to surge secretion of GnRH in the central nervous system (14). It is reported that peripheral (plasma) metastin levels are increased by pregnancy (15). Although a relationship between central and peripheral metastin has not been established, aging or gender can change movement of central and/or peripheral metastin. In the current study, however, there was no relationship between age and plasma metastin-LI levels (Fig. 1) and no significant difference between female and male in healthy individuals and in patients (Fig. 3).

Interestingly, we first showed that the plasma metastin-LI levels in pancreatic cancer patients were significantly higher when compared to healthy subjects who were of a similar age (Fig. 3), indicating that pancreatic cancer tissue has an influence on the level of plasma metastin-LI. However, plasma metastin-LI levels did not correlate with several tumor markers in cancer patients (Fig. 2). Although we did not measure the tumor marker levels in healthy subjects, the plasma metastin-LI levels might correlate with the tumor markers if we could add the data from non-cancer subjects.

Since the pancreas is located deep inside the body, the diagnosis of cancer is made by imaging such as computerized tomography or echography, but the tumor biopsy is sometimes very difficult. Furthermore, symptoms that are recognized by the patient, such as abdominal pain, jaundice, weight loss and gastrointestinal dysfunction may not appear until the cancer has progressed greatly. If the measurement of plasma metastin level enables the prediction of presence of pancreatic cancer, it may be of great help to diagnose the disease at an early stage.

The results of the current study suggest that the plasma metastin-LI of pancreatic cancer patients possibly is not a predictive indicator for tumor progression, invasion and lymph node and distant metastases (Figs. 4 and 5). In addition, there were no significant differences in the plasma metastin-LI levels in resectable and unresectable pancreatic cancer patients, although the metastin-LI levels in unresectable patients were significantly different from healthy volunteers. Further study will be necessary to establish cut-off values to predict extrapancreatic progression and/or resectable status of this disease. 
It is important to investigate whether metastin could be a biomarker to predict recurrence of pancreatic cancer and/or efficacy of treatments.

It is known that almost all cancer tissues, as well as pancreatic cancer, do not overexpress metastin, when compared with normal tissues and metastin has a metastasissuppressive effect (6). Although the amount of KiSS-1 does not always reflect plasma metastin levels, it is difficult to imagine that cancer tissue secretes a substance which stops their progression. A plausible explanation for the high level of plasma metastin-LI in cancer patients is that metastin may be secreted from normal tissue as a reaction to cancer progression, which is a self-defense mechanism. There have been no studies that measured plasma metastin levels in other kind of cancer, therefore, it is not known whether all kinds of cancer show such a profile as plasma metastin levels directly proportional to cancer progression. Gender-hormonedependent cancer, such as breast and prostate cancers, may have quite a different aspect.

Although further prospective study is needed, the measurement of plasma metastin levels and investigation of the relationship between plasma metastin and clinical cancer stage is important, not only to reveal the physiological action of metastin, but also to evaluate its effectiveness as a biomarker. Metastin could become a biomarker to predict cancer existence and its progression.

\section{References}

1. Muller A, Homey B, Soto H, et al: Involvement of chemokine receptors in breast cancer metastasis. Nature 410: 50-56, 2001.

2. Lee JH and Welch DR: Identification of highly expressed genes in metastasis-suppressed chromosome 6/human malignant melanoma hybrid cells using subtractive hybridization and differential display. Int J Cancer 71: 1035-1044, 1997.

3. Ikeguchi M, Hirooka $Y$ and Kaibara N: Quantitative reverse transcriptase polymerase chain reaction analysis for KiSS-1 and orphan G-protein-coupled receptor (hOT7T175) gene expression in hepatocellular carcinoma. J Cancer Res Clin Oncol 129: $531-535,2003$
4. Ringel MD, Hardy E, Bernet VJ, Burch HB, Schuppert F, Burman KD and Saji M: Metastin receptor is overexpressed in papillary thyroid cancer and activates MAP kinase in thyroid cancer cells. J Clin Endocrinol Metab 87: 2399, 2002.

5. Martin TA, Watkins G and Jiang WG: KiSS-1 expression in human breast cancer. Clin Exp Metastasis 22: 503-511, 2005.

6. Ohtaki T, Shintani Y, Honda S, et al: Metastasis suppressor gene KiSS-1 encodes peptide ligand of a G-protein-coupled receptor. Nature 411: 613-617, 2001.

7. Ikeguchi M, Yamaguchi K and Kaibara N: Clinical significance of the loss of KiSS-1 and orphan G-protein-coupled receptor (hOT7T175) gene expression in esophageal squamous cell carcinoma. Clin Cancer Res 10: 1379-1383, 2004.

8. Sanchez-Carbayo M, Capodieci P and Cordon-Cardo C: Tumor suppressor role of KiSS-1 in bladder cancer: loss of KiSS-1 expression is associated with bladder cancer progression and clinical outcome. Am J Pathol 162: 609-617, 2003.

9. Masui T, Doi R, Mori T, et al: Metastin and its variant forms suppress migration of pancreatic cancer cells. Biochem Biophys Res Commun 315: 85-92, 2004.

10. Anonymous: Pancreas. In: TNM Classification of Malignant Tumours. Sobin L and WitteKind C (eds). Wiley-Lewis, New York, pp93-96, 2002.

11. Katagiri F, Tomita K, Oishi S, Takeyama M and Fujii N: Establishment and clinical application of enzyme immunoassays for determination of luteinizing hormone releasing hormone and metastin. J Pept Sci 13: 422-429, 2007.

12. Kitagawa T, Shimozono T, Aikawa T, Yoshida $\mathrm{T}$ and Nishimura H: Preparation and characterization of heterobifunctional cress-linking reagents for protein modifications. Chem Pharm Bull 29: 1130-1135, 1981

13. Dungan HM, Clifton DK and Steiner RA: Minireview: kisspeptin neurons as central processors in the regulation of gonadotropinreleasing hormone secretion. Endocrinology 147: 1154-1158, 2006.

14. Kinoshita M, Tsukamura H, Adachi S, et al: Involvement of central metastin in the regulation of preovulatory luteinizing hormone surge and estrous cyclicity in female rats. Endocrinology 146: 4431-4436, 2005

15. Horikoshi Y, Matsumoto H, Takatsu Y, Ohtaki T, Kitada C, Usuki S and Fujino M: Dramatic elevation of plasma metastin concentrations in human pregnancy: metastin as a novel placentaderived hormone in humans. J Clin Endocrinol Metab 88: 914-919, 2003 